\title{
Circumcision in the United States: medical and nonmedical attitudes
}

\author{
Arnold G. Coran \\ Section of Pediatric Surgery, University of Michigan Medical School and C. S. Mott Children's Hospital, Ann Arbor, \\ Michigan, USA
}

The issue of routine neonatal circumcision has continued to generate debate in the United States in recent years. In 1975, the Ad Hoc Task Force on Circumcision of the American Academy of Pediatrics concluded that there was no absolute medical indication for routine circumcision of the male newborn. This conclusion reconfirmed the findings of the Committee on Fetus and Newborn of the American Academy of Pediatrics in 1971. In the Task Force report, the various traditional arguments for circumcision such as prevention of phimosis, reduction of the incidence of carcinoma of the penis and carcinoma of the cervix, reduction of the incidence of carcinoma of the prostate, and elimination of balanitis were refuted. The 1975 Task Force felt that a program of education leading to continuing good personal hygiene would offer all the advantages of a routine circumcision without the attendant surgical risk. Specifically, proper penile hygiene appeared to be just as effective as circumcision in the prevention of carcinoma of the penis and cervix and in the elimination of balanitis [1]. However, during this same period, Burger and Guthrie presented a strong argument for routine neonatal circumcision shortly before the report of the Task Force [2]. These authors argued in favor of neonatal circumcision for the prevention of balanitis, phimosis and paraphimosis, carcinoma of the penis, and transmission of venereal diseases. In particular, they felt that the incidence of complications was so extremely low that the routine performance of this procedure was justified. These authors also felt that routine neonatal circumcision was reasonable from an economic point of view

Offprint requests to: A. G. Coran because the procedure does not require additional hospitalization and the surgical fee is minimal in comparison with circumcision performed in childhood or adult life. The report by Gee and Ansell demonstrating a very low complication rate with routine neonatal circumcision $(0.2 \%)$ lends further support to Burger and Guthrie's recommendations [3].

My own response to the report of the Task Force of the American Academy of Pediatrics and to Burger and Guthrie's article over the past 14 years has been to favor the approach taken by the American Academy of Pediatrics. I have never recommended routine neonatal circumcision to any parents. Nevertheless, I have found that most of the parents in my area of the United States elect to have a routine circumcision done on their male newborn. In my experience, this decision is unrelated to the educational level or degree of sophistication of the parents involved. In fact, in all cases I try to explain to the parents that there are no strong medical indications for circumcision; however, this information has seemed to have little impact on their decision. I have, therefore, allowed the parents to make the final decision, arguing strongly that the procedure, if desired, be done in the newborn period rather than at a later age. Therefore, it appears that most decisions for or against circumcision are emotional rather than rational on the part of the parents. This was substantiated in a recent study by Brown and Brown [4].

In this issue of Pediatric Surgery International, King et al. arrived at the same conclusion, showing that most mothers chose circumcision for their newborns for hygienic reasons and to have their sons have the same appearance as the father 
or spouse. In their article, they also state that the majority of physicians are against routine neonatal circumcision and that there are no good medical reasons to advocate this operation routinely in all newborn males. It is regarding this last point that I wish to elaborate further. Until 2 years ago, I would have completely agreed with all the conclusions drawn by King and his co-authors. However, the studies published by Wiswell et al. 1985, 1986, and 1987 have clearly shown a significant decrease (tenfold) in the incidence of urinary tract infections during the 1st year of life in male infants who had undergone routine neonatal circumcision [5-7]. King et al. have not referred to any of these studies in their article; this omission diminishes the validity of their conclusions. The studies by Wiswell et al. have changed my attitude toward neonatal circumcision recently.

In conclusion, the studies of Wiswell and his colleagues have prompted me to change my attitude toward routine neonatal circumcision and to recommend it for all newborn males, providing the procedure is carried out by an experienced surgeon. I would add one additional caveat to this saga, namely that the operation should be done utilizing a penile block with local anesthesia, which eliminates all the pain and discomfort associated with this procedure.

\section{References}

1. Report of the Ad Hoc Task Force on Circumcision (1975) Pediatrics 56:610-611

2. Burger R, Guthrie TH (1974) Why circumcision? Pediatrics 54: 362-364

3. Gee WF, Ansell JS (1976) Neonatal circumcision: a tenyear overview with comparison of the Gomco clamp and the plastibell device. Pediatrics 58: 824-827

4. Brown MS, Brown CA (1987) Circumcision decision: prominence of social concerns. Pediatrics $80: 215-219$

5. Wiswell TE, Smith FR, Bass JW (1985) Decreased incidence of urinary tract infections in circumcised male infants. Pediatrics 75: 901-903

6. Wiswell TE, Roscelli JD (1986) Corroborative evidence for the decreased incidence of urinary tract infections in circumcised male infants. Pediatrics 78: 96-99

7. Wiswell TE, Enzenauer RW, Holton ME, Cornish D, Han kins CT (1987) Declining Frequency of Circumcision: Implications for Changes in the Absolute Incidence and male to female sex ratio of urinary tract infections in early infancy. Pediatrics 79:338-342 